\title{
A Consistent Model for the Large-Scale Steady Surface Atmospheric Circulation in the Tropics*
}

\author{
David S. Battisti and E. S. SARAchiK \\ Department of Atmospheric Sciences, University of Washington, Seattle, Washington
}

A. C. HiRsT

CSIRO Division of Atmospheric Research, Aspendale, Victoria, Australia

(Manuscript received 12 June 1997, in final form 23 October 1997)

ABSTRACT

\begin{abstract}
The authors present a new model of the tropical surface circulation, forced by changes in sensible heat and evaporative flux anomalies that are associated with prescribed sea surface temperature anomalies. The model is similar to the Lindzen and Nigam (LN) boundary layer model, also driven by the above flux anomalies; but here, since the boundary layer is assumed well mixed and capped by an inversion, the model reduces to a twolayer, reduced-gravity system. Furthermore, the rate of exchange of mass across the boundary layer-free atmosphere interface is dependent on the moisture budget in the boundary layer. When moist convection is diagnosed to occur, detrainment operates on the timescale associated with the life cycle of deep convection, approximately eight hours. Otherwise, the detrainment is assumed to be associated with the mixing out of the stable tropical boundary layer, which has a timescale of about one day. The model provides a diagnostic estimate of the anomalies in precipitation. However, it is assumed that the latent heat is released above the boundary layer, and it drives a circulation that does not impact the boundary layer.

The authors discuss the derivations of the Gill-Zebiak (GZ) and Lindzen-Nigam models and highlight some apparent inconsistencies between their derivation and the values of several of the parameters that are required for these models to achieve realistic solutions for the circulations. Then, the new reduced-gravity boundary model equations are rewritten in the form of the GZ and LN models. Using realistic values for the parameters in the new model geometry, it is shown that the constants combine in the rewritten equations to produce the physically doubtful constants in the GZ and LN models, hence, the reason for the apparent success of these models.
\end{abstract}

\section{Introduction}

Over the last two decades, there were a considerable number of studies published in which investigators presented models of the steady tropical atmosphere response to sea surface temperature (SST) anomalies. These studies, summarized below, were primarily adapted from the classic work of Gill on the steady response of the tropical atmosphere to heating.

In his original paper, Gill (1980) proposed that the major features of the tropical atmospheric circulation could be explained by the linearized equations of mo-

\footnotetext{
* Joint Institute for the Study of the Atmosphere and Ocean Contribution Number 70 .
}

Corresponding author address: Dr. David S. Battisti, Department of Atmospheric Sciences, University of Washington, Box 351640, Seattle, WA 98195.

E-mail: david@atmos.washington.edu tion, damped by mechanical friction and radiational cooling, and forced by deep tropical (moist) convection (Fig. 1a). Furthermore, Gill assumed that the vertical distribution of the diabatic forcing was such that the atmosphere response was given by an assumed gravest baroclinic mode, confined to the troposphere. The equations for the Gill model reduce to that of a two-layer atmosphere driven by latent heat release $Q_{o}$ in convective towers, which is assumed to be distributed equally in each layer. The equations, written here for the surface layer, are

$$
\begin{aligned}
\epsilon_{D} \mathbf{u}+\beta \mathrm{y} \mathbf{k} \times \mathbf{u} & =-\boldsymbol{\nabla} \boldsymbol{\Phi}, \\
\epsilon_{D} \Phi+c_{a}^{2} \boldsymbol{\nabla} \cdot \mathbf{u} & =-\mathrm{Q}_{o},
\end{aligned}
$$

where $\mathbf{u}$ is the mass-weighted horizontal velocity averaged through the lower layer, $\Phi$ is the surface geopotential, $c_{a}$ is the atmospheric wave speed $\left(c_{a}=N D / \pi\right.$, where $N$ is the Brunt-Väisälä frequency and $D$ the troposphere thickness), $\beta y$ is the local Coriolis parameter, and $\epsilon_{D}^{-1}$ is the thermal and mechanical dissipation time. 
(a) Gill / Zebiak

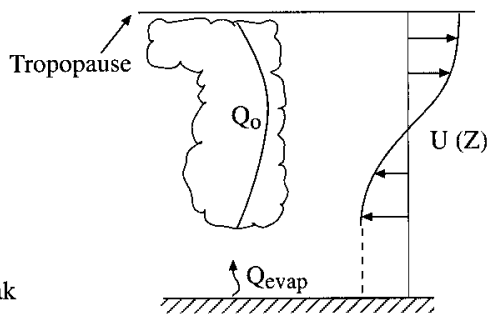

$$
\mathrm{Q}_{0}=\mathrm{Q}_{\text {evap }}+\mathrm{Q}_{\text {conv }}
$$

(b) Lindzen / Nigam

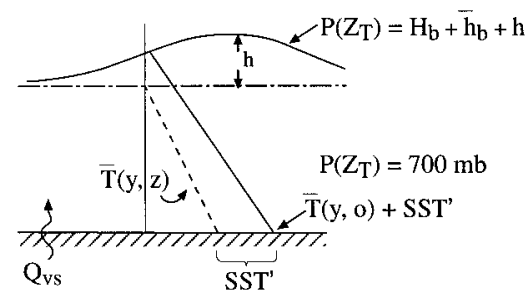

(c) Reduce Gravity

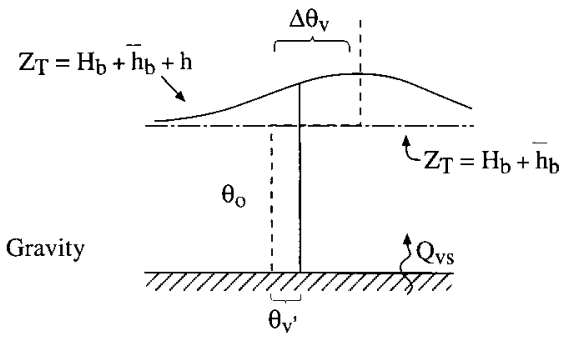

FIG. 1. Schematics of the steady-state atmosphere models discussed in this paper. (a) The Gill-Zebiak model (section 1). (b) The LindzenNigam model (section 2a). (c) The reduced gravity boundary layer model (sections $2 \mathrm{~b}$ and $2 \mathrm{c}$ ). The Gill model is a model of the circulation anomalies in the whole troposphere that are forced by anomalies in the diabatic heating (latent heat released) $Q_{o}$ in deep convective clouds; $Q_{o}$ has contributions from local evaporation $Q_{\text {evap }}$ and vertically integrated moisture convergence $Q_{\text {conv }}$. It is commonly assumed in the Gill-type models that the winds extend from the base of the (elevated) heating to the ground. The Lindzen-Nigam and RG models are models for boundary layer response to anomalies in sensible heat flux (or, more accurately, the virtual sensible heat flux $Q_{\mathrm{vs}}$ ) that results from changes in SST $\left(S S T^{\prime}\right){ }^{1}{ }^{1}$ The Lindzen-Nigam model assumes a linear vertical profile in basic state $(\bar{T})$ and anomalous $\left(T^{\prime}\right)$ temperature; air temperature anomalies at surface are equal to $S S T$ and linearly decay to zero at height $z=Z_{T}$ where $P\left(Z_{T}\right) \equiv 700 \mathrm{mb}$ In contrast, the RG boundary layer model assumes that the virtual sensible heat flux gives rise to virtual potential temperature anomalies that are well mixed in the boundary layer. The top of the boundary layer (at $z=Z_{T}$ ) is determined by hydrostatic effects and mixing across the boundary layer; the boundary layer is capped by a potentia temperature jump of $\Delta \theta_{v}$. In the Lindzen-Nigam (RG) models, the climatological mean height of the 700-mb surface (inversion) is at $z$ $=Z_{T}=H_{b}+\bar{h}_{b}$.

The variables $\mathbf{u}, \Phi$, and $Q_{o}$ are taken to be perturbations about a prescribed state.

\footnotetext{
${ }^{1}$ The virtual sensible heat flux $Q_{\mathrm{vs}}$ is the weighted sum of the sensible $Q_{s}$ and evaporative $Q_{\text {evap }}$ surface heat flux, which is equal to the pure sensible heat flux required to change the boundary layer
}

Investigators subsequent to Gill used this model, or modifications of this model, to examine the effects of SST anomalies in the Pacific Ocean during the El NiñoSouthern Oscillation (hereafter ENSO) on the surface winds (e.g., Gill and Rasmusson 1984; Zebiak 1986; Tian 1988). Gill and Rasmusson (1984) used the horizontal pattern of the outgoing longwave radiative anomaly from the 1982/83 ENSO event as a proxy for heating $Q_{o}$ and compared the model response to the observed circulation anomalies at $850 \mathrm{mb}$ in the tropical Pacific. They noted that the pattern of the response was consistent with the observed circulation pattern.

Gill and Rasmusson also noted that the convective (heating) anomalies were intimately tied to SST anomalies (see also Webster 1981). However, the prescription of $Q_{o}$ from SSTs is not obvious, since in the Gill model the moist convective forcing modifies the surface winds, whose convergence determines the heating. Zebiak (1986) explicitly included this effect by breaking up the heating $Q_{o}$ into an evaporative component, $Q_{\text {evap }}$, and a CISK-like component resulting from a change in the surface mass convergence, $Q_{\text {conv }}$, due to the anomalous surface circulation:

$$
Q_{o}=Q_{\text {evap }}\left(T^{\prime}, \bar{T}, \mathrm{rh}\right)+Q_{\text {conv }}\left(\bar{q}, \mathbf{u}^{\prime}, \overline{\mathbf{u}}\right),
$$

where $q$ is the mixing ratio, rh is the relative humidity (assumed constant), $T$ and $\mathbf{u}$ are surface temperature and wind, respectively, and overbars and primes denote climatological means and anomalies, respectively. Equations (1)-(3) are then solved by iteration. Zebiak (1986) presented the surface circulation that resulted when the Rasmusson and Carpenter (1982, hereafter RC) composite ENSO SST anomaly was used to calculate $Q_{\text {evap }}$ and the Gill model was forced by $Q_{o}$ as defined in Eq. (3). He compared the model-produced surface winds to the RC composite ENSO anomalies and noted that the convergence feedback term improved the amplitude and pattern of the model response. Tian (1988) and Kleeman (1991) modified $Q_{\text {conv }}$ to incorporate the effects of nonuniform fields of surface moisture convergence, $Q_{\text {conv }}^{\prime}\left[(q \mathbf{u})^{\prime}, \overline{q \mathbf{u}}\right]$, rather than mass convergence. These authors found the model circulation anomalies to be moderately improved over that forced by mass convergence. Davey and Gill (1987) used the depth-integrated moisture equation to determine $Q_{o}$ from an estimated precipitation rate.

Despite the overall success of the Gill model in reproducing the surface circulation anomalies during ENSO, some fundamental problems are known to exist with the model's formulation. As pointed out by Sardeshmukh and Hoskins (1985), the upper-level vorticity balance in the Tropics is inherently nonlinear. The Gill model is applied to the upper-level flow and is linear;

\footnotetext{
virtual temperature at the same rate. It may be easily shown that $Q_{\mathrm{vs}}$
} $=Q_{s}+\left(a c_{p} / L\right) Q_{\text {evap }}$ where $a=0.6 \bar{T} \simeq 180 \mathrm{~K}$. 
it cannot reproduce this vorticity balance. Another problem with the Gill model is the need for an extremely short damping time (1-2 days) to horizontally confine the response (realistic damping affords a global response to isolated heating) and produce realistic meridional winds. It is also well known that the heating due to penetrative moist convection does not extend to the surface. Thus, the surface circulation should be extremely sensitive to the cloud-base level (Wang 1988), and the lower level wind must exhibit large shear. ${ }^{2}$ The decoupling of the observed surface and $850-\mathrm{mb}$ flow field anomalies during ENSO is documented by Deser (1989).

There appears to be another problem with the Gill model, one that is ultimately related to the intricate coupling between the surface circulation and the upper-level circulation. A reexamination of the scaling of Zebiak's evaporation term $Q_{o}$ in Eq. (3) has revealed that, in this model, surface latent heating anomalies from evaporation are in excess of $75 \mathrm{~W} \mathrm{~m}^{-2}$ for a $1^{\circ} \mathrm{C}$ temperature anomaly. The model flux anomalies, averaged over a typical ENSO event, are four to five times larger than those observed for an SST anomaly of this size (see, e.g., Deser 1989). When the Gill model is driven by more typical values of $Q_{0}$, the resulting surface circulation anomalies forced by SST anomalies are insignificant. Similarly, Kleeman (1991) found that the local evaporation anomalies yielded only a small fraction of the total tropospheric heating in a model where the positioning of the deep convection was governed mainly by moist static energy and moisture convergence.

Together, these problems force us to conclude that the Gill model, when conceived of as a model for tropospheric-scale circulations, cannot be used to generically simulate the tropical tropospheric circulation anomalies, especially when forced indirectly via surface heat fluxes.

In another pioneering study, Lindzen and Nigam (1987; hereafter LN) have taken an alternative approach to modeling the tropical surface circulation anomalies. They assume SST anomalies produce sensible heat and evaporative fluxes, which, in turn, produce virtual temperature anomalies, $T_{v}^{\prime}(x, y)$, that are rapidly mixed into a confined boundary layer, producing surface pressure gradients that drive a surface circulation (Fig. 1b). They further assume that any surface convergence is everywhere rapidly vented by convection: LN set thermal damping time, $\boldsymbol{\epsilon}_{T}^{-1}$, to be $30 \mathrm{~min}$ based on the approximate timescale for the development of convection. An implicit assumption in the LN model, and contrary to the Gill model, is that the upper-level deep convection plays a minor role in forcing the low-level wind field.

\footnotetext{
${ }^{2}$ The circulation in the Tropics is constrained through a required balance between vertical motion and diabatic heating only when the heating is strong, for example, in moist convective regions (Holton 1992). Pressure gradient forces can significantly modify this balance in weakly diabatic regions.
}

Instead, the boundary layer anchors the location of the deep convection to the underlying SST anomalies, and the upper level only acts as a reservoir for the loweratmosphere heat and momentum perturbations.

While we basically agree with the LN model, there are some fundamental problems with the model. First, there are large regions of the Tropics and subtropics that rarely experience deep convection. Where the tropical atmosphere is not typically characterized as undergoing deep convection (e.g., throughout the subsiding subtropics and along the east Pacific cold tongue), the boundary layer should relax on a timescale associated with mechanical damping or entrainment, which occurs on timescales on the order of $\epsilon_{D}^{-1} \simeq 1$ day (Schubert et al. 1979). Second, Neelin (1989) has shown that the boundary layer convergence in the LN model, which is indicative of moist convective anomalies in the $\mathrm{LN}$ formulation, is proportional to the SST anomaly. However, the observed SST anomalies are not well correlated with anomalies in precipitation (see, e.g., Deser 1989). Finally, the adjustment time $\epsilon_{T}^{-1}$ required for the LN model to achieve physically realistic solutions is $O(30 \mathrm{~min})$, which we believe is unjustifiably short. For quasi-equilibrium solutions, the timescale associated with the large-scale relaxation of the tropical boundary layer that is characterized by deep convection is the timescale associated with the life cycle of the convective mixing of the boundary layer, which is approximately $8-12 \mathrm{~h}$ (e.g., Bretherton et al. 1995). In regions of strong subsidence, the adjustment timescale is approximately 1 day and is determined by entrainment and mixing processes across the top of the PBL (Schubert et al. 1979).

In this article, we will incorporate the effects of the density discontinuity at the top of the boundary layer on the thermally induced pressure anomaly within the boundary layer. In this way, physically realistic and consistent solutions will be obtained and the inconsistencies noted above in previous models will be reconciled.

The article proceeds as follows. In section 2, we briefly review the model of LN and develop a modified reduced-gravity model based on a moisture budget criterion. Also in this section is a brief discussion of the relationship between the depth-integrated transport and the surface winds in this model. A critique of the new model in relation to previous studies is presented in section 3. A summary and discussion are presented in section 4 .

\section{The model development}

\section{a. The Lindzen and Nigam boundary layer model}

Lindzen and Nigam, in developing their boundary layer model, noted the following. Over the oceans, the lower tropical troposphere is usually well mixed due to buoyant convection originating from the surface. They argued that the mixed layer, which includes the surface layer and in places the stratocumulus-cumulus cloud 
layer, extends to typically 2 or $3 \mathrm{~km}$ where it is capped by a strong temperature inversion brought about by subsiding, drier air (see, e.g., Riehl 1979; Sarachik 1985), particularly in the regions of the undisturbed trades. Even on spatial scales that are large compared to those of deep convection, it is likely that the Tropics will also be characterized by a boundary layer capped by a substantial inversion.

Lindzen and Nigam, using the First Global Atmospheric Research Program (GARP) Global Experiment (FGGE) data, further noted that the eddy temperature field, $T_{v}^{\prime}(x, y)$, defined as the deviation from the zonal mean, is vertically well correlated in the lower troposphere below $700 \mathrm{mb}$. Hence, they assumed the following simple expression for the eddy temperature field in the lower troposphere:

$$
T(x, y, z)=\bar{T}(y, z)+T_{v}^{\prime}(x, y)\left(1-\frac{\gamma}{H_{b}} z\right),
$$

with

$$
\bar{T}(y, z)=\bar{T}_{s}-\alpha z
$$

where $\bar{T}_{s}$ is the undisturbed zonal mean surface temperature, $\alpha$ is the undisturbed lapse rate, $H_{b}$ is a reference height (taken by LN to be $3 \mathrm{~km}$, or the approximate height of the 700-mb surface), and $\gamma$ is an $O(1)$ constant controlling the diminution with the height of the expression of the surface eddy temperature field. Here, as in LN, all temperatures refer to the virtual temperature.

In the LN model the eddy temperature field gives rise to a pressure gradient that defines the surface layer circulation. The horizontal momentum equation for the mass-weighted average flow in the boundary layer is

$$
\epsilon_{D} \mathbf{U}_{b}+\beta y \mathbf{k} \times \mathbf{U}_{b}=-\nabla \Phi_{\mathrm{LN}},
$$

where $\epsilon_{D}$ is now the mechanical dissipation (or mixing) rate throughout the boundary layer, and $\mathbf{U}_{b}$ the massweighted average boundary layer perturbation transport,

$$
\mathbf{U}_{b} \equiv \frac{1}{Z_{T} \rho\left(Z_{T}\right)} \int_{0}^{Z_{T}} \rho \mathbf{u} d z \simeq \frac{1}{H_{b} \rho\left(H_{b}\right)} \int_{0}^{H_{b}} \rho \mathbf{u} d z .
$$

The top of the boundary layer (taken by LN to be the 700-mb surface) is at $Z_{T}=H_{b}+\overline{h_{b}}+h$, where $\overline{h_{b}}$ is the zonal mean deviation of the boundary layer top from the reference height $H_{b}$, and $h$ the remaining "eddy" perturbation in the boundary layer height (see Fig. 1b). (Note, $|h| / H_{b},\left|h / \overline{h_{b}}\right|$ and $\left|\overline{h_{b}}\right| / H_{b}$ are taken as small.)

The perturbation (eddy) pressure gradient averaged over the depth of the boundary layer from the surface $(z=0)$ to the $Z_{T}$ is written [see LN, their Eqs. (6c) and (7c)]

$$
\frac{1}{Z_{T} \rho\left(Z_{T}\right)} \int_{0}^{Z_{T}} \nabla P d z \simeq \nabla \Phi_{\mathrm{LN}}
$$

where

$$
\Phi_{\mathrm{LN}} \equiv g\left(1+\alpha H_{b} / \bar{T}_{s}\right) h-\Gamma_{\mathrm{LN}} T_{v}^{\prime}(x, y),
$$

and $\Gamma_{\mathrm{LN}}=\left(g H_{b} / 2 \bar{T}_{s}\right)(1-2 \gamma / 3)$ and $g$ is the gravitational acceleration. The two contributions to the perturbation pressure are the changing thickness of the boundary layer due to fluctuations at the top $(h)$ and changes in the density of the air in the boundary layer due to thermal perturbations, $T_{v}^{\prime}(x, y)$.

Lindzen and Nigam argued that in regions of deep convection the surface mass convergence would be rapidly vented by convection. Therefore, according to (9), the full effects of the hydrostatically induced pressure gradient would not be realized. They incorporated this "back pressure" effect through the vertically averaged continuity equation and further assumed the boundary layer could relax back to equilibrium in time $\epsilon_{T}^{-1}$ :

$$
-H_{b} \boldsymbol{\nabla} \cdot \mathbf{U}_{b} \simeq w\left(Z_{T}\right)=\epsilon_{T} h .
$$

In the steady state, the velocity at the top of the boundary layer is the entrainment velocity, taken proportional to the boundary layer perturbation height (mass convergence). ${ }^{3}$

Neelin (1989) noted the mathematical similarity of the Gill model [Eqs. (1) and (2)] to the LN model by rewriting Eq. (9) using (10) whereby the LN model equations become

$$
\begin{aligned}
\epsilon_{D} \mathbf{U}_{b}+\beta y \mathbf{k} \times \mathbf{U}_{b} & =-\nabla \Phi_{\mathrm{LN}}, \\
\epsilon_{D} \Phi_{\mathrm{LN}}+C_{\mathrm{LN}}^{2} \boldsymbol{\nabla} \cdot \mathbf{U}_{b} & =-\epsilon_{D} \Gamma_{\mathrm{LN}} T_{v}^{\prime}(x, y),
\end{aligned}
$$

where

$$
C_{\mathrm{LN}}^{2}=g H_{b}\left(1+\frac{\alpha H_{b}}{\bar{T}_{s}}\right) \frac{\epsilon_{D}}{\epsilon_{T}} \simeq g H_{b} \frac{\epsilon_{D}}{\epsilon_{T}} \equiv C_{B}^{2} \frac{\epsilon_{D}}{\epsilon_{T}} .
$$

The mathematical similarity between the LN model [Eqs. (6) and (11)] and the Gill model [Eqs. (1) and (2)] is now apparent, although the physics is fundamentally different. Further discussion is deferred until section 3 .

\section{b. The reduced-gravity boundary layer model ( $R G)$}

We hereby adopt the physically intuitive geometry of a well-mixed boundary layer of constant virtual potential temperature:

$$
\begin{gathered}
\Theta_{v}(x, y)=\Theta_{o}+\bar{\Theta}_{v}(x, y)+\Theta_{v}^{\prime}(x, y) \\
\text { for } 0<z<Z_{T},
\end{gathered}
$$

where $\Theta_{o}$ is a reference temperature, and $\bar{\Theta}_{v}$ and $\Theta_{v}^{\prime}(x, y)$ are the basic state and perturbation virtual potential temperature in the boundary layer, respectively. The top of the boundary layer will be of variable depth,

$$
Z_{T}=H_{b}+\overline{h_{b}}+h
$$

\footnotetext{
${ }^{3}$ Hence, radiation is assumed to have a timescale that is longer than that of entrainment or mixing.
} 
and capped by an unperturbed constant inversion of strength $\Delta \Theta_{v}$. The model is shown schematically in Fig. 1c. The notation in Eq. (14) is as in the LN model, except the top of the boundary layer (at $z=Z_{T}$ ) is now defined by the level at which the inversion is found rather than by the level of a constant pressure surface. The boundary layer includes the mixed layer and, when present, the stratocumulus-cumulus cloud layer. There is abundant observational evidence for this model geometry in the tropical and subtropical marine environment (see, e.g., Riehl 1954, 1979; Augstein et al. 1974; Stage and Businger 1981; Nicholls and Leighton 1986), including the regions near the intertropical convergence zones (Bunker 1971; Ramage et al. 1981).

Following LN, we will assume that the boundary layer flow is driven directly by hydrostatically induced pressure gradients that are associated with the perturbations in the virtual potential temperature, $\Theta_{v}^{\prime}(x, y)$, which are confined to be within the well-mixed boundary layer. Thus, we assume that the role of the free atmosphere (above $Z_{T}$ ) is to reduce gravity and absorb mass and heat from the boundary layer without producing significant vertically averaged flow throughout the troposphere above the boundary layer. (Significant baroclinic flow above the boundary layer can result from deep convection, but we assume that the integrated effects of this flow at the top of the boundary layer are small compared to the boundary layer processes. ${ }^{4}$ )

We obtain, by a mass-weighted average of the momentum equation for the boundary layer, the equation for the surface flow:

$$
\epsilon_{D} \mathbf{U}_{b}+\beta y \mathbf{k} \times \mathbf{U}_{b}=-\nabla \Phi_{\mathrm{RG}},
$$

where $\mathbf{U}_{b}$ is defined in Eq. (7) and $\Phi_{\mathrm{RG}}$ is the perturbation pressure for the reduced gravity (RG) system:

$$
\Phi_{\mathrm{RG}} \equiv g^{\prime} h-\Gamma \Theta_{v}^{\prime}(x, y),
$$

where $g^{\prime} \equiv g \Delta \Theta_{v} / \Theta_{o}$ is the value of reduced gravity and $\Gamma=g H_{b} / 2 \Theta_{o}$ [terms of $O\left(|h| / H_{b},\left|\Theta_{v}^{\prime}(x, y)\right| / \Delta \Theta_{v}\right)$ have been neglected in Eq. (16)]. Following LN, we will assume that, in the steady state, the rate of relaxation of the boundary layer perturbations will depend on the mass convergence in the boundary layer. The relaxation rate, however, will now depend on whether or not there is enough moisture to support convection. Since we are interested in the equilibrium solution, when convection (above the boundary layer) is diagnosed, the venting of the boundary layer will be taken as the timescale $\epsilon_{M}^{-1}$ associated with the life cycle of deep convection..$^{5}$ In the absence of deep convection, the mixing out

\footnotetext{
${ }^{4}$ The model formulation is familiar to oceanographers as a $1 \frac{1}{2}$ layer model forced by bouyancy rather than wind stress.

${ }^{5}$ Note that the physics involved in the parameter $\epsilon_{M}$ is identical to $\epsilon_{T}$ used by LN. We distinguish the two by the numerical values assigned to this process, which differ by a factor of 16 (48) if deep convection is diagnosed (is not diagnosed) to be occurring.
}

of the boundary layer perturbations will be at the slower entrainment rate, $\epsilon_{D}$, observed to be $O[1 /(1-2$ days $)]$ (see section 1). Hence, the equations for the reducedgravity model of the tropical boundary layer flow driven by hydrostatically induced pressure gradients are [cf. Eqs. (6), (11), and (12)]

$$
\epsilon_{D} \mathbf{U}_{b}+\beta y \mathbf{k} \times \mathbf{U}_{b}=-\nabla \Phi_{\mathrm{RG}}
$$

convecting,

$$
\epsilon_{D} \Phi_{\mathrm{RG}}+\frac{\epsilon_{D}}{\epsilon_{M}} C_{\mathrm{RG}}^{2} \boldsymbol{\nabla} \cdot \mathbf{U}_{b}=-\epsilon_{D} \Gamma \Theta_{v}^{\prime}(x, y),
$$

not convecting,

$$
\epsilon_{D} \Phi_{\mathrm{RG}}+C_{\mathrm{RG}}^{2} \boldsymbol{\nabla} \cdot \mathbf{U}_{b}=-\epsilon_{D} \Gamma \Theta_{v}^{\prime}(x, y),
$$

where

$$
C_{\mathrm{RG}}^{2} \equiv g^{\prime} H_{b} \equiv C_{B}^{2} \frac{\Delta \Theta_{v}}{\Theta_{o}} .
$$

It will prove to be convenient to write (17) and (18) as one equation:

$$
\epsilon_{D} \Phi_{\mathrm{RG}}+C_{\mathrm{RG}}^{2}\left(1-\beta^{\prime}\right) \boldsymbol{\nabla} \cdot \mathbf{U}_{b}=-\epsilon_{D} \Gamma \Theta_{v}^{\prime}(x, y),
$$

where

$$
\beta^{\prime} \equiv\left\{\begin{array}{ll}
1-\epsilon_{D} \epsilon_{M}^{-1} & \text { if convecting } \\
0 & \text { otherwise }
\end{array}\right\} .
$$

The primary differences between the boundary layer model presented here and the original LN boundary layer model are as follows: (i) the effect of gravity is reduced from that in $\mathrm{LN}$ by formulating a two-layer system, and (ii) the boundary layer relaxation rate depends on the convective activity above the boundary layer. The latter effect acts to stiffen the boundary layer where convection is not occurring $\left(\beta^{\prime}=2 / 3\right)$, hence producing convergence and pressure perturbations of smaller amplitude and greater horizontal extent.

\section{c. The precipitation criterion and venting rate}

It remains for us to ascertain whether the boundary layer circulation is supporting convection. We stress that, in the LN and RG models, the convective process only acts to restore the boundary layer flow: diabatic heating via precipitation does not originate from within the boundary layer. In the steady state, precipitation must occur if the evaporation rate exceeds the moisture divergence due to the flow field. Since evaporation is at the sea surface and most of the moisture is found within the boundary layer, a diagnostic check for precipitation is provided by the moisture budget (see, e.g., Weare 1986):

$$
\begin{aligned}
P \equiv & \left.\rho_{\text {air }} C_{E}|\mathbf{u}|[q(S S T)-q(\text { air })]\right|_{z=0} \\
& -\int_{0}^{z_{T}} \boldsymbol{\nabla} \cdot\left(\rho_{\text {air }} q(\text { air }) \mathbf{u}\right) d z-\delta_{m},
\end{aligned}
$$


TABLE 1. Values for the constants used in various steady-state tropical atmosphere models. The values that are in bold are qualitatively inconsistent with the observations. The * indicates a wave speed given by Eq. (12). The typical forcing values denote anomalies that are observed during a standard deviation one ENSO.

\begin{tabular}{|c|c|c|c|c|c|}
\hline Model type & Model source & $\begin{array}{l}\text { Mechanical } \\
\text { damping } \\
\epsilon_{\mathrm{D}}^{-1} \text { (days) }\end{array}$ & $\begin{array}{c}\text { Venting or } \\
\text { thermal } \\
\text { damping } \\
\epsilon_{\mathrm{T}}^{-1}\end{array}$ & $\begin{array}{c}\text { Wave speed } \\
\mathrm{m} \mathrm{s}^{-1}\end{array}$ & $\begin{array}{l}\text { Typical } \\
\text { forcing } \\
\left(W m^{-2}\right)\end{array}$ \\
\hline $\begin{array}{l}\text { Convective (full } \\
\text { troposphere) }\end{array}$ & $\begin{array}{l}\text { Gill-Zebiak } \\
\text { observations }\end{array}$ & $\begin{aligned} & \mathbf{1 - 2} \\
\approx & 10\end{aligned}$ & $\begin{array}{l}\text { 1-2 days } \\
\approx 15 \text { days }\end{array}$ & $\begin{aligned} & 60 \\
\approx & 40\end{aligned}$ & $\begin{array}{l}75 \\
15-20 \quad \text { (evaporative) }\end{array}$ \\
\hline Boundary layer & $\begin{array}{l}\text { Lindzen-Nigam } \\
\text { Reduced gravity } \\
\quad \text { (section 2b) } \\
\text { Observations }\end{array}$ & $\begin{array}{l}\approx 1-2 \\
\approx 1-2 \\
\approx 1-2\end{array}$ & $\begin{aligned} & 30 \mathrm{~min} \\
& 8 \mathrm{~h} \\
\approx & 3-12 \mathrm{~h}\end{aligned}$ & $\begin{array}{l}\approx 18 \\
\approx 18\end{array}$ & $\begin{array}{l}\text { 5-10 (virtual sensible) } \\
\text { 5-10 (virtual sensible) } \\
5-10 \text { (virtual sensible) }\end{array}$ \\
\hline
\end{tabular}

where the bulk formula for evaporation has been assumed and $P \geq 0$. In Eq. (22), $\delta_{m}$ is the loss of moisture from the boundary layer to the free atmosphere by ubiquitous mixing (detrainment).

If the circulation is found to support convection $(P$ $>0$ ), rapid venting is assumed for the boundary layer and Eq. (17) applies. However, if Eq. (22) indicates a net moisture deficit in the boundary layer $(P<0)$, then there is no rain in the steady state, and the restoration of the boundary layer circulation is by the relatively inefficient process of mixing across the inversion (entrainment): Eq. (18) applies. In practice, the model equations (20), (21), and (22) are solved iteratively.

\section{d. The surface winds}

The boundary layer model presented in section $2 \mathrm{~b}$ is for the mass transport averaged over the depth of the boundary layer. Neelin (1989) showed that the momentum equations (15) are a good approximation for the integrated boundary layer transport in the Geophysical Fluid Dynamics Laboratory GCM and, hence, provide a reasonable estimate of the moisture convergence in the boundary layer. Nonetheless, the holy grail for investigators that use the steady (slave) atmospheric models is frequently the surface wind velocity. To this end, we note that the empirical results of Deser (1993) could be used in conjunction with the reduced-gravity model to give a more reasonable solution for the surface winds, once the transport averaged over the boundary layer is determined via Eqs. (15)-(18).

\section{Critique of simple tropical atmosphere models}

A large body of literature has developed concerning large-scale atmosphere-ocean instability that is based on the atmospheric model formulated by Gill (1980) (see, e.g., Philander et al. 1984; Rennick 1983; Gill 1985; Hirst 1986). This model atmosphere formulation is also utilized in most studies that have led to the formulation of the potential mechanisms for the ENSO phenomenon and interannual variability in the Tropics (see, e.g., Anderson and McCreary 1985; Zebiak and Cane 1987; Schopf and Suarez 1988; Battisti 1988; Bat- tisti and Hirst 1989; Wakata and Sarachik 1991). Yet the cumulative problems with the Gill model, noted in section 1 and summarized in Table 1, suggest this framework must be considered to have only qualitative value (see also Seager 1991).

The formulation of the boundary layer model of LN, on the other hand, is somewhat more consistent with the observations. The same mathematical equations are realized in both models [cf. Eqs. (1) with (6), and (2) with (11)], except the boundary layer model yields the flow averaged in the surface layer rather than the baroclinic tropospheric flow field. Neelin (1988) has presented calculations that indicate that the momentum equation (6) forced by the 700-mb vertical velocity from a GCM is indeed a good approximation to the flow over the ocean in the lowest $300 \mathrm{mb}$ of a general circulation model with damping times $\epsilon_{D}^{-1} \simeq 2$ days. Zebiak (1990) has presented similar conclusions based on an analysis of observed flow and pressure fields. This suggests that linear dynamics may be sufficient for the boundary layer flow. In Neelin's (1988) calculation, the forcing at the top of the boundary layer [effectively the $\Phi_{\mathrm{LN}}$ term in Eq. (6)] was prescribed from the GCM calculations. In general, the perturbation height will be due to heating of the boundary layer from surface fluxes, vertical profile of radiation flux, turbulent entrainment of dry air from above into the boundary layer, and detrainment from the top of the boundary layer, the latter being sensitive to the presence of convection aloft.

The heating of the Gill model by Zebiak's (1986) iterative forcing scheme Eq. (3) includes a convective heating term that is proportional to the SST anomaly, and a term that depends on the total (mass) convergence in the boundary layer. Zebiak's heating scheme can be approximated as follows (see Zebiak 1985):

$$
\underset{\text { total heating }}{Q_{o}}=\underset{(\mathrm{A})}{\Lambda T^{\prime}}-\beta_{Z} c_{a}^{2} \boldsymbol{\nabla} \cdot \mathbf{u},
$$

where

$$
\beta_{Z}= \begin{cases}3 / 4 & \text { convecting } \\ 0 & \text { not convecting. }\end{cases}
$$

Hereafter, the Gill model [Eqs. (1) and (2)] forced by 
Zebiak's formulation of heating [Eq. (23)] will be referred to as the Gill-Zebiak (GZ) model.

The term (A) is the evaporatively induced convective heating anomaly, while (B) is a CISK-like feedback term. Zebiak noted that the magnitude of the phase speed $\left(c_{a}^{2}, C_{\mathrm{LN}}^{2}\right)$ in the Gill and $\mathrm{LN}$ models is very similar, and the forcing functions [convective $\Lambda T^{\prime}$, evaporative $\left.\epsilon_{D} \Gamma T_{v}^{\prime}(x, y)\right]$ have similar dependence on SST anomalies and amplitude $\left[T_{v}^{\prime}(x, y) \simeq \mathrm{SST}^{\prime}\right]$. Zebiak (1990) further demonstrated that including the CISK term (B) in the heating of the Gill model yields surface circulation and pressure distributions more like the observations.

The generally good simulation of the surface flow field provided by the physically problematic Gill model (see Table 1 and section 1), when forced by Zebiak's (1986) heating scheme, can now be understood by contrasting the set of equations for the Gill model [with heating via Eq. (23)] with the equations for the RG boundary layer model presented in section $2 \mathrm{~b}$ :

Gill-Zebiak model

$$
\begin{aligned}
\underline{\epsilon_{D} \mathbf{u}}+\beta y \mathbf{k} \times \mathbf{u} & =-\boldsymbol{\nabla} \Phi, \\
\underline{\epsilon_{D} \Phi}+c_{a}^{2} \boldsymbol{\nabla} \cdot \mathbf{u} & =-\underline{\Lambda T^{\prime}}+\beta_{Z} c_{a}^{2} \boldsymbol{\nabla} \cdot \mathbf{u},
\end{aligned}
$$

RG boundary layer model

$$
\begin{aligned}
\epsilon_{D} \mathbf{U}_{b}+\beta y \mathbf{k} \times \mathbf{U}_{b}= & -\nabla \Phi_{\mathrm{RG}}, \\
\epsilon_{D} \Phi_{\mathrm{RG}}+C_{\mathrm{RG}}^{2} \boldsymbol{\nabla} \cdot \mathbf{U}_{b}= & -\epsilon_{D} \Gamma \Theta_{v}^{\prime}(x, y) \\
& +\beta^{\prime} C_{\mathrm{RG}}^{2} \boldsymbol{\nabla} \cdot \mathbf{U}_{b} .
\end{aligned}
$$

[Equation (24) is from Eqs. (1) and (23); Eq. (25) is Eq. (20) rewritten.] The mathematical formulation of both models is identical; there are minor differences on how convection $\left(\beta_{z} ; \beta^{\prime}\right)$ is diagnosed. In addition, the values of the key parameter combinations are similar:

Gill model

$$
\begin{aligned}
& \left.\begin{array}{l}
c_{a}=60 \mathrm{~m} \mathrm{~s}^{-1} \\
\Lambda=2.5 \times 10^{-3} \mathrm{~m}^{2} \mathrm{~s}^{-3} \mathrm{~K}^{-1}
\end{array}\right\} \\
& \Rightarrow \boldsymbol{\nabla} \cdot \mathbf{u} \simeq \frac{\Lambda}{c_{a}^{2}} T^{\prime}=7 \times 10^{-7} \mathrm{~s}^{-1} \mathrm{~K}^{-1},
\end{aligned}
$$

RG boundary layer model

$$
\begin{aligned}
& \left.\begin{array}{rl}
C_{\mathrm{RG}}=17.9 \mathrm{~m} \mathrm{~s}^{-1} \\
\epsilon_{D} \Gamma=6.4 & \times 10^{-4} \mathrm{~m}^{2} \mathrm{~s}^{-3} \mathrm{~K}^{-1}
\end{array}\right\} \\
& \Rightarrow \nabla \cdot \mathbf{U}_{b} \simeq \frac{\epsilon_{D} \Gamma}{C_{\mathrm{RG}}^{2}} \Theta_{v}^{\prime}=1.95 \times 10^{-6} \mathrm{~s}^{-1} \mathrm{~K}^{-1} .
\end{aligned}
$$

Hence, it is to be expected that the two models yield similar answers. However, we noted in section 1 that some fundamental assumptions made in the Gill formulation are inconsistent with the observed atmospheric flow (e.g., the inherent nonlinearity in the upper tropical tropospheric vorticity balance and, in the eastern and central equatorial Pacific, the decoupling of the surface and 850 -mb flow fields). Additionally, some of the values required for the parameters in the Gill and GZ models are unrealistic: these terms are underlined in Eqs. (1) and (24) above and summarized with the observed values in Table 1.

Turning now to the LN model, we note that the extremely short venting time $\epsilon_{T}$, required therein for realistic flow fields, is essentially compensated for in the present RG model by the hitherto neglected reducedgravity effect (see Table 1). Indeed, we expect a similar response of the two boundary layer models in convective regions, based on the comparison of the thermodynamic equations (11) and (20) (the momentum equation in the two models is identical). The only difference between the models is in the "effective" phase speeds:

$$
\frac{C_{\mathrm{LN}}^{2}}{C_{\mathrm{RG}}^{2}}=\frac{\epsilon_{D} / \epsilon_{T}}{\left(1-\beta^{\prime}\right) \Delta \Theta_{v} / \Theta_{o}}=O(1) .
$$

This article is limited to a discussion of concept. Some calculations were made with the RG model, using a range of specified SST anomaly patterns for the tropical Pacific (D. Battisti 1990, unpublished manuscript). The results were found to be generally similar to those previously calculated by LN and by Zebiak (1986), as may be expected from the similar form and coefficient values of the resulting models. The point here is that the form and values may be obtained via the reduced-gravity boundary layer model, without recourse to physically doubtful values for the forcing or damping coefficients.

Recently, Wang and Li (1993) also pointed out the deficiencies in the Gill-Zebiak and LN model formulations and showed that a combined approach involving a baroclinic mode free atmosphere interacting with a planetary boundary layer was able to provide a broadly realistic surface wind pattern using realistic values of model parameters connected to boundary layer venting and thermal damping. However, they had to retain an extremely strong Rayleigh friction (approximately 1 day $^{-1}$ ) for the baroclinic mode free atmosphere in order to obtain realistic solutions. Moreover, Wang and $\mathrm{Li}$ (1993) took the top of the boundary layer to be a fixed isobaric surface and found that their solution was very sensitive to the assumed depth of this boundary layer. Kleeman (1991) was able to obtain fairly realistic boundary layer winds with a linear three-pressure-level atmospheric model with somewhat simpler boundary layer physics than that of Wang and Li (1993). Like Wang and Li (1993), Kleeman took the top of the boundary layer to be a fixed isobaric surface; thus, he also had to prescribe a very strong Rayleigh friction for all three model layers. When the boundary layer is instead treated as a variable-depth layer subject to reduced gravity, we argue the bulk of the boundary layer flow can be accounted for without the explicit baroclinic free atmosphere component with its problematic damping coefficients.

Finally, Yu and Neelin (1997) have presented a linear 
steady tropical atmosphere model that, in some respects, is complementary to our model. The former model develops a thermodynamically consistent framework for determining the vertical structure of the free tropospheric response to changes in surface boundary conditions, and it is valid only where the atmosphere is undergoing deep moist convection. In contrast, our model assumes a priori that a boundary layer exists and highlights how changes in the surface SST boundary conditions affect the surface circulation.

\section{Conclusions}

We review in this article two popular models for the response of the tropical atmosphere to prescribed tropical SST anomalies: the Gill model (as modified by Zebiak 1986) and the Lindzen and Nigam (1987) model, referred to as the GZ and LN models, respectively. The GZ model assumes that the SST drives a deep circulation in the tropical troposphere through changes in the surface evaporation. The anomalies in the surface winds result explicitly from changes in deep convection that are assumed to be driven by changes in the SST. In contrast, the LN model assumes the surface wind anomalies are entirely due to changes in the boundary layer virtual temperature distribution resulting from evaporative and sensible heat flux anomalies associated with SST anomalies. Changes in deep convection act only indirectly to change the surface circulation by "venting" the boundary layer so as to reduce the hydrostatically induced horizontal pressure gradients. Unfortunately, both the GZ and LN models obtain apparently realistic solutions for the surface winds only if physically doubtful values are assigned to several of the parameters therein.

We overcome this problem of model derivation by introducing a new model of the steady tropical surface circulation. The model is similar to the LN, only here the boundary layer is assumed to be well mixed, to have variable depth, and to be capped by an inversion. Hence, the model reduces to a two-layer, reduced-gravity system. The rate of exchange of mass across the boundary layer-free atmosphere interface is dependent on the moisture budget in the boundary layer. Because we are finding the equilibrium solution, when convection is diagnosed to occur, detrainment operates on the timescale associated with the life cycle of deep convection (approximately $8-12 \mathrm{~h}$ ). Otherwise, the detrainment timescale is assumed to be associated with the mixing out of the stable tropical boundary layer (approximately 1 or 2 days). The reduced-gravity boundary layer model provides a diagnostic estimate of the anomalies in precipitation, though the latent heat released is above the boundary layer and is assumed to drive a circulation that does not impact the boundary layer.

Finally, we rewrite the reduced-gravity boundary layer model equations in the form of the GZ and LN models. Using realistic values for the parameters in the new model configuration, we show that the constants combine in the rewritten equations to produce the physically doubtful constants in the GZ and LN models, hence the reasons for the apparent success of these models.

Postscript. This paper was written in 1990. We had intended to augment the work presented here with a section on the role of the Andes (which eliminates some, but not all, of the "excessive easterly" problems in the Gill model) and with extensive calculations. Though we lost interest in the work and did not complete these sections, the work that we did complete is requested (and cited) regularly enough that we feel obligated to publish the completed portions of the work. For the many people who have a copy of the original manuscript, the only changes in the published version of the paper are (i) a short discussion in section 3 of the models by Wang and Li (1993) and Yu and Neelin (1997), which had not been developed when we wrote the original manuscript; and (ii) a discussion in section $2 \mathrm{~d}$ of the surface winds as a function of the integrated mass transport in the boundary layer, which is inspired by the work of Deser (1993).

Acknowledgments. We are grateful for conversations with Steve Zebiak and Richard Seager during the course of this work (in 1990). We thank the anonymous reviewers who provided us with very constructive and thoughtful comments that led to improvements in our paper. This work was partially funded by the Joint Institute for the Study of the Atmosphere and Ocean under NOAA Cooperative Agreement NA67RJ0155 and a grant from the NOAA Office of Global Programs to the Hayes Center.

\section{REFERENCES}

Anderson, D., and J. P. McCreary, 1985: Slowly propagating disturbances in a coupled ocean-atmosphere model. J. Atmos. Sci., 42, 615-629.

Augstein, E., H. Schmidt, and F. Ostapoff, 1974: The vertical structure of the atmospheric boundary layer in undisturbed trade winds over the Atlantic Ocean. Bound.-Layer Meteor., 6, 129-150.

Battisti, D. S., 1988: Dynamics and thermodynamics of a warming event in a coupled tropical atmosphere-ocean model. J. Atmos. Sci., 45, 2889-2919.

- , and A. C. Hirst, 1989: Interannual variability in a tropical atmosphere-ocean model: Influence of the basic state, ocean geometry and nonlinearity. J. Atmos. Sci., 46, 1687-1712.

Bretherton, C. S., P. Austin, and S. T. Siems, 1995: Cloudiness and marine boundary layer dynamics in the ASTEX Lagrangian experiments. Part II: Cloudiness, drizzle, surface fluxes, and entrainment. J. Atmos. Sci., 52, 2724-2735.

Bunker, A. F., 1971: Energy transfer and tropical cell structure over the central Pacific. J. Atmos. Sci., 28, 1101-1116.

Davey, M. K., and A. Gill, 1987: Experiments on tropical circulation with a simple moist model. Quart. J. Roy. Meteor. Soc., 113, 1237-1269.

Deser, C., 1989: Meteorogical characteristics of the El Niño/Southern Oscillation phenomenon. Ph.D. thesis, University of Washington, $195 \mathrm{pp}$.

- 1993: Diagnosis of the surface momentum balance over the tropical pacific ocean. J. Climate, 6, 64-74. 
Gill, A. E., 1980: Some simple solutions for heat induced tropical circulation. Quart. J. Roy. Meteor. Soc., 106, 447-462.

- 1985: Elements of coupled ocean-atmosphere models for the tropics. Coupled Ocean-Atmosphere Models, J. Nihoul, Ed., Elsevier Oceanography Series, Vol. 40, Elsevier, 303-327.

— equatorial Pacific. Nature, 306, 229-234.

Hirst, A. C., 1986: Unstable and damped equatorial modes in simple coupled models. J. Atmos. Sci., 43, 606-630.

Holton, J. R., 1992: An Introduction to Dynamic Meteorology. Academic Press, 507 pp.

Kleeman, R., 1991: A simple model of the atmospheric response to ENSO sea surface temperature anomalies. J. Atmos. Sci., 48, 318.

Lindzen, R. S., and S. Nigam, 1987: On the role of sea surface temperature gradients in forcing low-level winds and convergence in the Tropics. J. Atmos. Sci., 44, 2418-2436.

Neelin, J. D., 1988: A simple model for the surface stress and low level flow in the tropical atmosphere driven by prescribed heating. Quart. J. Roy. Meteor. Soc., 114, 747-770.

, 1989: On the interpretation of the Gill model. J. Atmos. Sci., 46, 2466-2468.

Nicholls, S., and J. Leighton, 1986: An observational study of the structure of stratiform cloud sheets. Part I. Structure. Quart. J. Roy. Meteor. Soc., 112, 431-460.

Philander, S., T. Yamagata, and R. Pacanowski, 1984: Unstable airsea interactions in the Tropics. J. Atmos. Sci., 41, 604-613.

Ramage, C., S. Khalsa, and B. Meisner, 1981: The central Pacific near-equatorial convergence zone. J. Geophys. Res., 86, 65806598.

Rasmusson, E. M., and T. H. Carpenter, 1982: Variations in tropical sea surface temperature and surface wind fields associated with the Southern Oscillation/El Niño. Mon. Wea. Rev., 110, 354384.

Rennick, M. A., 1983: A model of atmosphere-ocean coupling in El Niño. Trop. Ocean-Atmos. Newsl., 15, 2-4.

Riehl, H., 1954: Tropical Meteorology. McGraw-Hill, 392 pp. , 1979: The Trade Wind Inversion. Academic Press, $611 \mathrm{pp}$.

Sarachik, E. S., 1985: A simple theory for the vertical structure of the tropical atmosphere. Pure Appl. Geophys., 123, 261-271.

Sardeshmukh, P. D., and B. Hoskins, 1985: Vorticity balances in the
Tropics during the 1982-1983 El Niño-Southern Oscillation event. Quart. J. Roy. Meteor. Soc., 111, 261-278.

Schopf, P. S., and M. J. Suarez, 1988: Vacillations in a coupled oceanatmosphere model. J. Atmos. Sci., 45, 549-566.

Schubert, W. H., J. S. Wakefield, E. J. Steiner, and S. K. Cox, 1979: Marine stratocumulus convection. Part II: Horizontally inhomogeneous solutions. J. Atmos. Sci., 36, 1308-1324.

Seager, R., 1991: A simple model of the climatology and variability of the low-level wind field in the Tropics. J. Climate, 4, 164179.

Stage, S. A., and J. Businger, 1981: A model for entrainment in a cloud-topped marine boundary layer. Part I: Model description and application to a cold-air outbreak. J. Atmos. Sci., 38, 22132229.

Tian, P., 1988: A consistent model for the response of the atmosphere to sea surface temperature anomaly during El Niño. M.S. thesis, Dept. of Atmospheric Sciences, University of Washington, 149 pp.

Wakata, Y., and E. S. Sarachik, 1991: Unstable coupled atmosphereocean basin modes in the presence of a spatially varying basic state. J. Atmos. Sci., 48, 2060-2077.

Wang, B., 1988: Dynamics of tropical low-frequency waves: An analysis of the moist Kelvin wave. J. Atmos. Sci., 45, 2051-2065.

_, and T. Li, 1993: A simple tropical atmosphere model of relevance to short-term climate variations. J. Atmos. Sci., 50, 260284.

Weare, B. C., 1986: A simple model of the tropical atmosphere with circulation dependent heating and specific humidity. J. Atmos. Sci., 43, 2001-2016.

Webster, P. S., 1981: Mechanisms determining the atmospheric response to sea surface temperature anomalies. J. Atmos. Sci., 38, 554-571.

Yu, J. Y., and J. D. Neelin, 1997: Analytic approximations for moist convectively adjusted regions. J. Atmos. Sci., 54, 1054-1063.

Zebiak, S. E., 1985: Tropical atmosphere-ocean interaction and the El Niño/Southern Oscillation phenomenon. Ph.D. thesis, Massachusetts Institute of Technology, $261 \mathrm{pp}$.

- 1986: Atmospheric convergence feedback in a simple model for El Niño. Mon. Wea. Rev., 114, 1263-1271.

1990: Diagnostic studies of Pacific surface winds. J. Climate, 3, 1016-1031.

, and M. Cane, 1987: A model El Niño/Southern Oscillation. Mon. Wea. Rev., 115, 2262-2278. 